\title{
Shape control of FePt nanocrystals
}

\author{
Narayan Poudyal, Girija S. Chaubey, Chuan-bing Rong, and J. Ping Liu ${ }^{\text {a) }}$ \\ Department of Physics, University of Texas at Arlington, Arlington, Texas 76019, USA
}

(Presented 14 November 2008; received 17 September 2008; accepted 5 January 2009; published online 6 April 2009)

\begin{abstract}
FePt nanocrystals were prepared by simultaneous reduction of platinum acetylacetonate and thermal decomposition of iron pentacarbonyl in properly chosen solvents/surfactants. A variety of FePt nanocrystals, including nanowires, nanorods, nanocubes, and nanosized multipods, were successfully obtained. The shape control was realized by simply varying synthesis parameters. The as-synthesized nanocrystals, having chemically disordered fcc structure, are found to be superparamagnetic at room temperature and their ferromagnetic-superparamagnetic transition temperature is size dependent that increases with particle size. The as-synthesized FePt nanocrystals were transformed to the chemically ordered $L 1_{0}$ structure upon heat treatment, which exhibits hard magnetic properties with coercivity as high as $25 \mathrm{kOe}$. () 2009 American Institute of Physics. [DOI: 10.1063/1.3077210]
\end{abstract}

\section{INTRODUCTION}

Synthesis of nanocrystals with different sizes and shapes has opened new technological horizons. ${ }^{1,2}$ Since it has been observed that physical and chemical properties of nanoparticles are also related to their shape, synthesis of nanocrystals with controllable shape has become a recent focus. ${ }^{3,4}$ Arranging differently shaped nanocrystals into ordered patterns with specific packing arrangement by self-assembly or other techniques is an important step toward design of novel nanostructured materials and devices. ${ }^{5,6}$ In particular, FePt nanocrystals have been widely studied because of their great application potential in advanced magnetic materials such as ultrahigh-density recording media and high-performance permanent magnets, based on their uniaxial magnetocrystalline anisotropy. ${ }^{7-10}$ Recent progress in chemical synthesis techniques for preparing FePt nanoparticles has shown a great deal of success in tuning particle size while maintaining a narrow size distribution. ${ }^{11-13}$ Progress has also been made in controlling shape of FePt nanoparticles, including preparation of one-dimensional structures such as nanorods and nanowires. ${ }^{14-16}$ We recently reported that simultaneous reduction in platinum acetylacetonate $\left[\mathrm{Pt}(\mathrm{acac})_{2}\right]$ and iron pentacarbonyl $\left(\mathrm{Fe}(\mathrm{CO})_{5}\right)$ in the presence of 1,2-hexadecanediol (HDD), oleylamine, and oleic acid in a high-boiling ether solvent leads to the formation of $\mathrm{FePt}$ nanorods and nanowires. ${ }^{14}$

Further experiments indicated that shape of the $\mathrm{FePt}$ nanocrystals can be conveniently controlled through one-pot reactions without any ether solvent but just surfactants. In this report we give details of this novel facile method for synthesizing FePt nanocrystals with controlled shape and dimension, including nanowires, nanorods, spherical nanoparticles, nanocubes, and nanosized multipods.

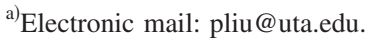

\section{EXPERIMENTS}

FePt nanocrystals were grown by the simultaneous reduction of $\mathrm{Pt}(\mathrm{acac})_{2}$ and the thermal decomposition of $\mathrm{Fe}(\mathrm{CO})_{5}$ in the presence of HDD, oleylamine, and oleic acid. We have established a new method for controlling the shape of FePt nanocrystals by controlling the reaction kinetics, the molar ratio of the surfactants, the addition of sequence of surfactants, and the reaction temperatures.

In a typical recipe of synthesis of FePt nanowires, a mixture of $0.25 \mathrm{mmol} \mathrm{Pt}(\mathrm{acac})_{2}$ and $100 \mathrm{mg}$ of HDD was added to a $125 \mathrm{ml}$ European flask containing a magnetic stir bar. $10 \mathrm{ml}$ of oleylamine was then injected into the flask and the contents were stirred while purging with $\mathrm{N}_{2}$ for $20 \mathrm{~min}$ at room temperature. The flask was then heated to $100{ }^{\circ} \mathrm{C}$ and the temperature was held for $20 \mathrm{~min}$. During this time, 0.05 mmol of $\mathrm{Fe}(\mathrm{CO})_{5}$ was injected into the flask while $\mathrm{N}_{2}$ purging was continued. After that, $2 \mathrm{ml}$ of oleic acid was injected and the mixture was maintained under $\mathrm{N}_{2}$ blanket and heated to $300{ }^{\circ} \mathrm{C}$ at a rate of $1.5^{\circ} \mathrm{C} / \mathrm{min}$. The mixture was maintained at the refluxing temperature of $300{ }^{\circ} \mathrm{C}$ for $30 \mathrm{~min}$ before cooling down to room temperature under the $\mathrm{N}_{2}$ blanket. The length of FePt nanorods/nanowires can be controlled from 20 to over $150 \mathrm{~nm}$ by varying the molar concentration of HDD and oleic acid while keeping other parameters constant. For example, FePt nanowires with length over $150 \mathrm{~nm}$ were formed when $100 \mathrm{mg}$ of HDD and $2 \mathrm{ml}$ of oleic acid were used. While $300 \mathrm{mg}$ of HDD was taken with $2 \mathrm{ml}$ of oleic acid, the length of wires was reduced to $20 \mathrm{~nm}$. The diameter of the FePt nanorods can also be tuned from 3 to $5 \mathrm{~nm}$ by altering the molar ratio of oleylamine and oleic acid from 2:1 to 2:1.5. Further increases of HDD and oleic acid led to the formation of oval-shaped nanoparticles. When both the surfactants were added at the beginning of the reaction and the molar ratios of oleylamine to oleic acid were varied between 3:1 and 1:1, spherical nanoparticles, nanocubes, and multipods were formed (see details in Sec. III). Morphology, structure, composition and magnetic property of samples were studied using transmis- 


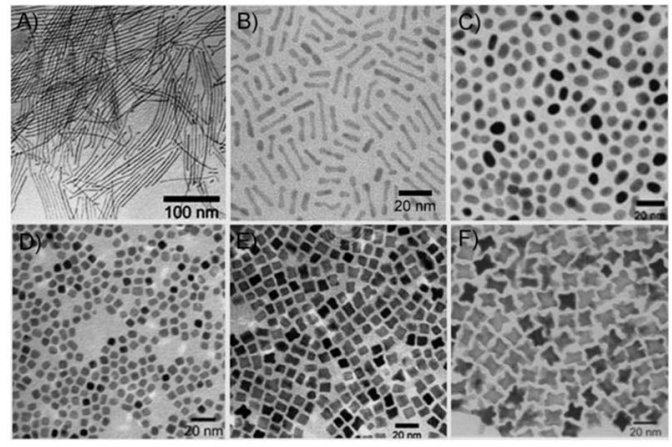

FIG. 1. TEM images of FePt with various shapes: (A) nanowires $(150 \pm 20 \mathrm{~nm} \times 3 \pm 0.5 \mathrm{~nm}),(B)$ nanorods $(20 \pm 5 \mathrm{~nm} \times 3 \pm 0.5 \mathrm{~nm}),(C)$ oval-shaped nanoparticles $(8 \pm 1.5 \mathrm{~nm})$, (D) spherical nanoparticles $(8 \pm 0.8 \mathrm{~nm})$, (E) nanocubes $(9 \pm 0.9 \mathrm{~nm})$, and $(F)$ nanosized multipods $(18 \pm 1.5 \mathrm{~nm})$.

sion electron microscopy (TEM), x-ray diffraction (XRD), energy dispersive $\mathrm{X}$-ray spectroscopy (EDX) and superconducting quantum interference device magnetometer (SQUID).

\section{RESULTS AND DISCUSSION}

Figure 1 shows TEM images of monolayer assemblies of the as-synthesized FePt nanocrystals with different morphologies, including nanowires, nanorods, oval-shaped particles, spherical particles, nanocubes, and multipods. It is known that surfactants play a vital role in growth of nanocrystals. ${ }^{3,7,9,11-14}$ The anisotropic interaction between the capping agents and the different facets of $\mathrm{Fe}-\mathrm{Pt}$ crystals should be the key for the formation of differently shaped nanocrystals. ${ }^{3}$ Typically, when a smaller amount of surfactants is injected simultaneously after the addition of $\mathrm{Fe}(\mathrm{CO})_{5}$ in the ether solvent, spherical FePt nanoparticles are formed. ${ }^{7,11-13}$ It has been found from our study that not only the molar ratio of surfactants but also the sequence of addition of the surfactants and metal precursors has influence on the shape of FePt nanoparticles. For example, nanorods and nanowires were obtained when oleylamine was added before the addition of $\mathrm{Fe}(\mathrm{CO})_{5}$, followed by oleic acid, at the molar ratio of oleylamine to oleic acid at 4:1[Figs. 1(A) and 1(B)]. Spherical particles were formed when both the surfactants were added simultaneously before the addition of $\mathrm{Fe}(\mathrm{CO})_{5}$, with molar ratio of oleylamine to oleic acid at 4:1 [Fig. 1(D)]. Under the similar reaction conditions as the synthesis of spherical particles, the nanocubes and the multipods were obtained [Figs. 1(E) and 1(F)], when the molar ratio of oleylamine to oleic acid was varied from $2: 1$ to $1: 1$. The as-

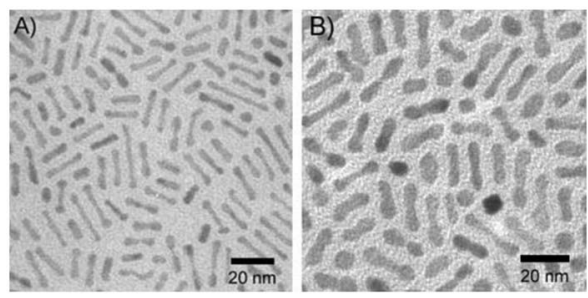

FIG. 2. TEM images of $20 \mathrm{~nm}$ long FePt nanorods with diameters of (A) 3 $\mathrm{nm}$ and (B) $5 \mathrm{~nm}$.

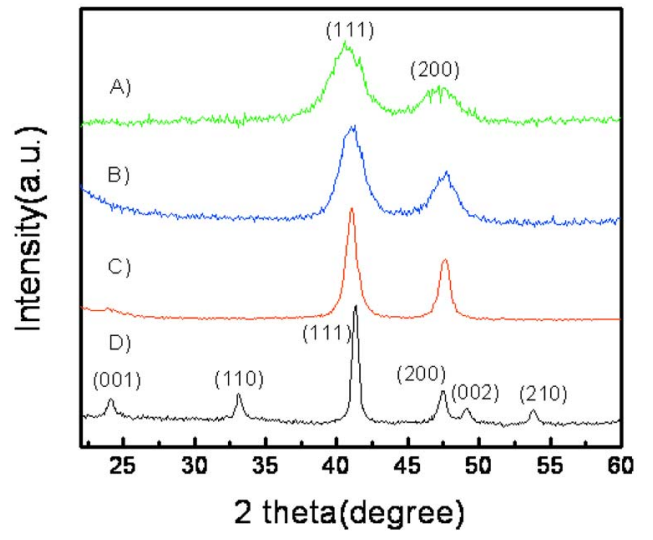

FIG. 3. (Color online) XRD patterns of as-synthesized FePt (A) nanowires, (B) spherical nanoparticles, (C) multipods, and (D) annealed FePt nanowires under forming gas $\left(\mathrm{Ar} 93 \%+\mathrm{H}_{2} 7 \%\right)$ at $700{ }^{\circ} \mathrm{C}$ for $1 \mathrm{~h}$.

synthesized FePt nanorods and nanowires [in Figs. 1(A) and 1(B)] have a diameter of $3 \mathrm{~nm}$ with lengths of about 20 and $150 \mathrm{~nm}$, respectively. The diameter of the FePt nanorods can be also tuned from 3 to $5 \mathrm{~nm}$ [Figs. 2(A) and 2(B)] by altering the molar ratio of oleylamine and oleic acid from 2:1 to 2:1.5 while other reaction parameters were kept unchanged. A further increase in HDD and oleic acid led to the formation of oval-shaped nanoparticles [Fig. 1(C)].

The XRD patterns in Figs. 3(A)-3(C) confirm that the as-synthesized FePt nanocrystals possess a chemically disordered fcc crystal structure, which has low magnetic anisotropy. It should be noted that XRD patterns show no preferred texture even for the one-dimensional nanocrystal assemblies deposited on Si substrates. The as-synthesized FePt nanocrystal assemblies are superparamagnetic at room temperature. Figure 4 shows the temperature dependent magnetization measured for nanocrystals in a 100 Oe field between 5 and $300 \mathrm{~K}$ with the zero-field-cooling (ZFC) and field-cooling (FC) procedures. On cooling, the ZFC magnetization begins to drop and deviates from FC magnetization at the blocking temperature. The blocking temperature is defined as $T_{B}=K V / 25 K_{B}$, where $K$ is the anisotropy energy constant, $V$ is the volume of the particles, and $K_{B}$ is the Boltzmann constant. ${ }^{19}$ The $M$ versus $T$ curves show a peak characteristic to a superparamagnetic transition. This obser-

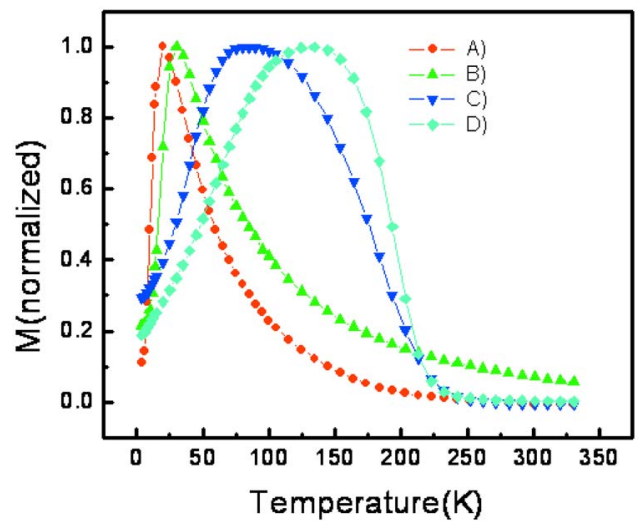

FIG. 4. (Color online) Magnetization vs temperature for as-synthesized FePt (A) nanorods, (B) nanowires, (C) spherical nanoparticles, and (D) multipod nanoparticles measured at 100 Oe field. 


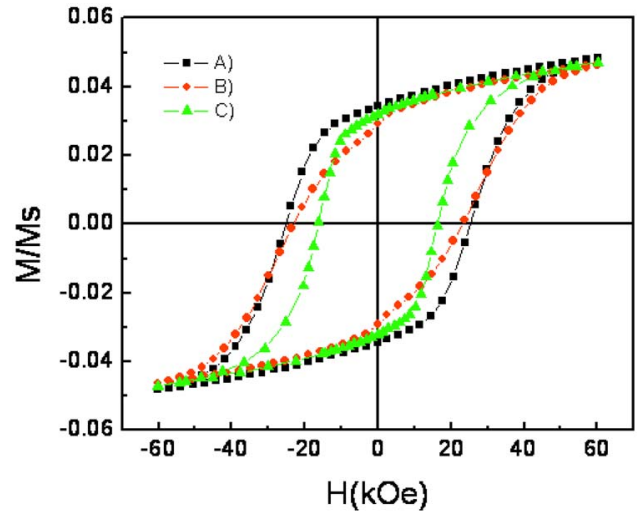

FIG. 5. (Color online) Hysteresis loops of FePt (A) nanowires, (B) multipods, and (C) spherical particles annealed in forming gas at $700{ }^{\circ} \mathrm{C}$ for $1 \mathrm{~h}$.

vation indicates that superparamagnetic behavior is blocked at $20,32,85$, and $134 \mathrm{~K}$ for $20 \mathrm{~nm}$ rods, $150 \mathrm{~nm}$ wires, $8 \mathrm{~nm}$ spherical particles, and $18 \mathrm{~nm}$ multipods, respectively (Fig. 4). The change in $T_{B}$ depends on the particle's volume, but the shape of the particles may also contribute to their anisotropy. In our study, differently shaped particles have different volumes. Therefore, it is hard to exactly estimate the contribution from the shape anisotropy to $T_{B}$ of the nanocrystals.

A heat treatment is needed to transform the disordered fcc FePt phase to the $L 1_{0}$ phase with high uniaxial magnetocrystalline anisotropy, which is desirable for applications. ${ }^{17}$ The as-synthesized FePt nanocrystal samples were annealed in forming gas at $700{ }^{\circ} \mathrm{C}$ for $1 \mathrm{~h}$. It was found that both the peak positions and intensities of the annealed samples match perfectly with the $L 1_{0}$ structure in XRD patterns. The heat treatments, however, also promote the sintering of $\mathrm{FePt}$ nanocrystals, which results in grain growth and a drastic change in morphology from discrete particles to continuous thin films. The average grain size calculated for annealed FePt nanowire assemblies, using the Scherrer formula, was $25 \mathrm{~nm}$ [Fig. 3(D)].

The hysteresis loops of randomly oriented assemblies of nanowires, multipods, and spherical nanoparticles annealed in forming gas at $700{ }^{\circ} \mathrm{C}$ for $1 \mathrm{~h}$ deposited on Si substrates are shown in Fig. 5. The high coercivity up to $25 \mathrm{kOe}$ of randomly oriented assemblies of nanocrystals annealed in forming gas at $700{ }^{\circ} \mathrm{C}$ indicates high magnetocrystalline anisotropy of the $L 1_{0} \mathrm{FePt}$ phase (in thin film morphology). However, differences in coercivity and the squareness of hysteresis loops for differently shaped nanocrystals are found but are not completely understood. Study with high resolution TEM on the morphology and microstructure of the annealed assemblies is underway. The compositions of the FePt nanocrystals were determined by EDX. Compositions of samples with high coercivity were found to be close to equiatomic. The final composition was controlled by changing the initial molar ratio of the metal precursors. Preparation of $L 1_{0}$ FePt nanocrystals with different shapes using salt- matrix annealing ${ }^{10,18,19}$ to prevent undesirable coalescing and sintering of the nanocrystals during heat treatment will be reported elsewhere.

\section{CONCLUSIONS}

In summary, a facile procedure has been developed for controlling the shape of FePt nanocrystals with a large variety of morphologies. The molar ratio of surfactants and the sequencing of the injection of the surfactants are the keys to controlling the reaction kinetics and anisotropic growth of the nanocrystals. The as-synthesized FePt nanocrystals have chemically disordered fcc structure and are superparamagnetic at room temperature. Their blocking temperatures increase with particle size. After the heat treatment the fcc structured FePt nanocrystals transformed into $L 1_{0}$ phase with coercivity up to $25 \mathrm{kOe}$. These nanocrystals with controlled geometry and composition can be used as building blocks for future ultrahigh-density recoding media, exchange-coupled nanocomposite permanent magnets, and other nanodevices.

\section{ACKNOWLEDGMENTS}

This work was supported by U.S. DoD/MURI Grant No. N00014-05-1-0497 and DARPA through ARO under Grant No. DAAD 19-03-1-0038. This work was also supported by Center of Nanostructured Materials and Characterization Center for Materials and Biology at the University of Texas at Arlington.

${ }^{1}$ A. I. Boukai, Y. Bunimovich, J. Tahir-Kheli, J.-K. Yu, W. A. Goddard III, and J. R. Heath, Nature (London) 451, 168 (2008).

${ }^{2}$ A. I. Hochbaum, R. Chen, R. D. Delgado, W. Liang, E. C. Garnett, M. Najarian, A. Majumdar, and P. Yang, Nature (London) 451, 163 (2008).

${ }^{3}$ X. Peng, L. Manna, W. Yang, J. Wickham, E. Scher, A. Kadavanich, and A. P. Alivisatos, Nature (London) 404, 59 (2000).

${ }^{4}$ L. Thomas, M. Hayashi, X. Jiang, R. Moriya, C. Rettner, and S. S. P. Parkin, Nature (London) 443, 197 (2006).

${ }^{5}$ E. V. Shevchenko, D. V. Talapin, N. A. Kotov, S. O'Brien, and C. B. Murray, Nature (London) 439, 55 (2006).

${ }^{6}$ C. T. Black, C. B. Murray, R. L. Sandstrom, and S. Sun, Science 290, $1131(2000)$

${ }^{7}$ S. Sun, C. B. Murray, D. Weller, L. Folks, and A. Moser, Science 287, 1989 (2000)

${ }^{8}$ H. Zeng, J. Li, J. P. Liu, Z. L. Wang, and S. Sun, Nature (London) 420, 395 (2002).

${ }^{9}$ S. Sun, Adv. Mater. (Weinheim, Ger.) 18, 393 (2006).

${ }^{10}$ C. B. Rong, D. R. Li, V. Nandwana, N. Poudyal, Y. Ding, Z. L. Wang, H. Zeng, and J. P. Liu, Adv. Mater. (Weinheim, Ger.) 18, 2984 (2006).

${ }^{11}$ K. E. Elkins, T. S. Vedantam, J. P. Liu, H. Zeng, S. Sun, Y. Ding, and Z. L. Wang, Nano Lett. 3, 1647 (2003).

${ }^{12}$ M. Chen, J. P. Liu, and S. Sun, J. Am. Chem. Soc. 126, 8394 (2004).

${ }^{13}$ V. Nandwana, K. E. Elkins, N. Poudyal, G. S. Chaubey, K. Yano, and J. P. Liu, J. Phys. Chem. C 111, 4185 (2007).

${ }^{14}$ N. Poudyal, G. S. Chaubey, V. Nandwana, C. B. Rong, K. Yano, and J. P. Liu, Nanotechnology 19, 355601 (2008).

${ }^{15}$ M. Chen, T. Pica, Y. Jiang, P. Li, K. Yano, J. P. Liu, A. K. Datye, and H. Fan, J. Am. Chem. Soc. 129, 6348 (2007).

${ }^{16}$ C. Wang, Y. Hou, J. Kim, and S. Sun, Angew. Chem., Int. Ed. 119, 6449 (2007).

${ }^{17}$ T. Klemmer, D. Hoydick, H. Okamura, B. Zhang, and W. A. Soff, Scr. Metall. Mater. 33, 1793 (1995).

${ }^{18}$ K. Elkins, D. Li, N. Poudyal, V. Nandwana, Z. Q. Jin, K. H. Chen, and J. P. Liu, J. Phys. D: Appl. Phys. 38, 2306 (2005).

${ }^{19}$ C. B. Rong, N. Poudyal, G. S. Chaubey, V. Nandwana, R. Skomski, Y. Q. Wu, M. J. Kramer, and J. P. Liu, J. Appl. Phys. 102, 043913 (2007). 